$\Rightarrow$

\title{
Reversing Huntington's disease
}

\section{ce}

The beneficial effects of ASO infusion on motor symptoms last longer than the effects of HTT suppression.
There are currently no effective disease-modifying treatments for Huntington's disease (HD), a hereditary neurodegenerative disorder in which expression of the mutant protein huntingtin (HTT) leads to a progressive decline in motor and cognitive function. Most investigative therapeutic approaches that aim to suppress the synthesis of mutant HTT have primarily targeted the striatum, the region of the brain that is most vulnerable to mutant HTT-mediated toxicity, but increasing evidence suggests that the targeting of multiple brain regions may be required for an effective therapy. Now, Kordasiewicz et al. show that transient infusion of single-stranded antisense oligonucleotides (ASOs) into the central nervous system of mouse models of HD leads to widespread suppression of HTT synthesis in the brain, resulting in sustained improvements in motor function and behaviour.

Using ASOs comprising a 20-mer phosphorothioate-modified oligonucleotide that is complementary to human HTT mRNA, the authors first showed that infusion of these ASOs for 2 weeks into the right lateral ventricle of the BACHD mouse model of HD suppressed mutant HTT expression in a dose-dependent manner for up to 12 weeks post-treatment. Moreover, the
ASOs were distributed to the neurons of most brain regions, including the frontal cortex, striatum, thalamus, midbrain, brainstem and cerebellum.

Next, they investigated the effects of this treatment regimen in the YAC128 and BACHD mouse models of HD. In both mouse models, ASO treatment led to improvements in motor activity and coordination, and hypoactivity and anxiety-like behaviour were ameliorated to levels similar to wild-type mice. In particular, for BACHD mice (which were followed up for 6 months longer than YAC128 mice), motor deficits were improved at up to 15 months of age, which is 9 months after treatment had stopped and more than 5 months after levels of mutant HTT expression returned to untreated levels. This indicates that the beneficial effects of ASO infusion on motor symptoms last longer than the effects of HTT suppression. Importantly, when levels of normal HTT were reduced by $75 \%$ in non-transgenic mice, through a mouse-targeted ASO, no changes in motor function were observed, thus showing that ASO-directed suppression of normal HTT is well tolerated.

Finally, intrathecal infusion into Rhesus monkeys of an ASO that is complementary to both Rhesus monkey and human HTT (for 21 days) showed that ASOs were

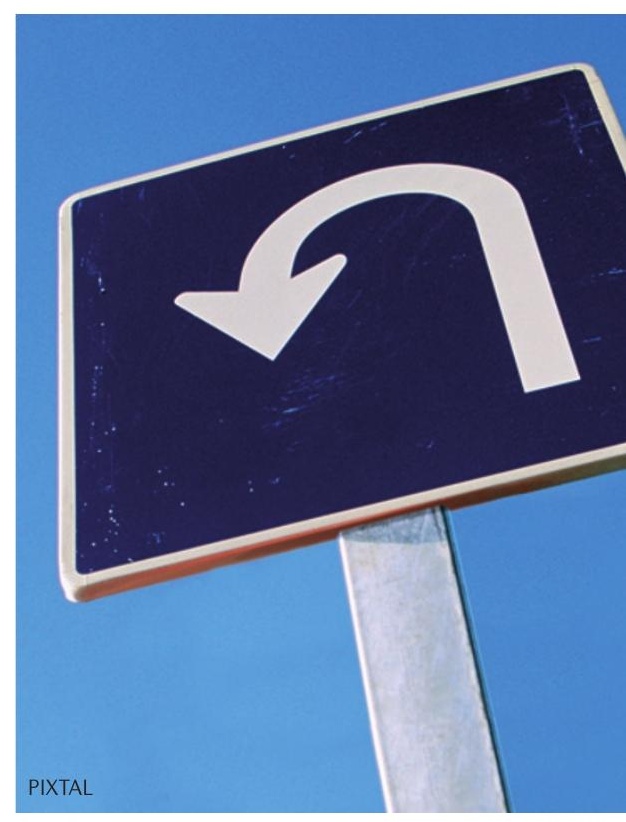

distributed to the neurons of most periventricular and lateral brain regions. HTT expression was significantly suppressed immediately after infusion and remained suppressed for 4 weeks after ending treatment.

Together, the authors suggest that this is a clinically viable approach to treat $\mathrm{HD}$, whereby a transient decrease in HTT synthesis could lead to a prolonged effect in patients.

\section{Man Tsuey Tse}

ORIGINAL RESEARCH PAPER Kordasiewicz, $H$. B. et al. Sustained therapeutic reversal of Huntington's disease by transient repression of huntingtin synthesis. Neuron 74, 1031-1044 (2012) 